\title{
BYRNE AND LONG: A CLASSIFICATION FOR RATING THE INTERVIEW STYLE OF DOCTORS
}

\author{
R. Buijs, E. M. Sluijs and P. F. M. VerhaAk \\ Netherlands Institute of General Practitioners, Department of Scientific Research, Postbox 812, \\ 3500 AV Utrecht, The Netherlands
}

\begin{abstract}
In order to assess the doctor's verbal behaviour in the consultation, a classification instrument is needed that will enable us to summarize the doctor's interview style. Such an instrument, developed by Byrne and Long, is evaluated in this study. After presenting the procedure as it has been worked out by Byrne and Long, some points of criticism are formulated. On account of our criticism, some changes have been introduced into the instrument. To test the relevance and reliability of the instrument, 36 consultations have been classified according to the adjusted system. This procedure showed that it was possibie to represent a consultation with the 45 categories the system consisted of (although only 15 categories occurred in more than half of the consultations). Those categories that occurred often enough, could be measured with sufficient reliability, with inter-observer reliability coefficients mostly above 0.70 . An example shows that the procedure is extremely useful to indicate certain differences between doctors and types of consultations.
\end{abstract}

\section{INTRODUCTION}

During consulting hours, general practitioners are confronted with a wide range of problems. As far as patient complaints have a purely organic ground, doctors tend to work with sufficient effectiveness. Their way of functioning, however, is more of a problem when they are offered mental complaints or physical complaints from a mental source. During their training most doctors have hardly or never been taught how to handle these. Some doctors try to compensate this deficiency by attending postgraduate courses or by consulting experts in this field. such as psychiatrists or psychologists.

Investigators, including those of The Netherlands Institute of General Practitioners (NHI), have been concerned with the question in how far this type of activity modifies the behaviour of doctors in their surgeries. One problem is that a measuring instrument to assess the doctor's verbal behaviour during the consultation does not yet exist. A measuring instrument is required, capable of summarizing the doctor's interview behaviour without violating the essence of a particular doctor's interview style. It will have to be an observation instrument since a precise representation of what doctors do is required rather than what they think they must do or believe they do in their consultations.

Some of the existing observation instrument leave out too much of the content of what is said in a consultation. We have in mind here observation systems that aim exclusively at the observation of non-verbal behaviour [1-3] and systems to which the function of the words spoken (offering, refusing, etc.) is central [4]. A system of categories as used in the Process Interaction Analysis of Bales, (cf. e.g. [5-9]) does deal with the content of an interview, but has in our opinion the drawback that the categories (e.g. 'asking questions' of 'problem solving') are too general (the entire system comprises some 10-14 categories). NHI systems of categories have up to now never been comprehensive enough to include every item of speech used in the consultation [10].

If we have the use of an instrument that would represent the whole consultation and also gives prominence to such aspects as particularly stand out in general practice, it is possible to name the differences between doctors, to trace changes in interview style and to give well-aimed feedback to doctors on their actions.

In their book Doctors Talking to Patients [11], Byrne and Long attempt to develop such an instrument. Below we wish to try and evaluate their work on its own merits. Besides a critical examination of their book we shall also present a few research data which are based on their ideas. Successively we offer here the procedure adopted by Byrne and Long and our criticisms of it. What is left of it after this critique will then be considered as to its permanent usefulness for the system.

\section{THE CLASSIFICATION SYSTEM OF BYRNE AND LONG}

In Doctors Talking to Patients Byrne and Long report on their study of the verbal behaviour of the GP during consulting hours. For this purpose they analysed over 2500 tape-recorded surgery interviews. Of these interviews each unit of sense was scored in any one of the 50 observation categories (see Appendix).

In this way an interview profile of a consultation could be devised on the basis of the incidence of the 50 observation categories. These interview profiles were not designed by Byrne and Long for the consultations as a whole; in every consultation they distinguished two phases, i.e. the 'diagnostic phase' and the 'prescribing phase'. The transition is when "the doctor appears to have made a decision about the condition of the patient" (p. 159). For the sake of clarity it must be noted that in one consultation the diagnostic phase and prescribing phase can alternate 
more than once, especially when the patient offers several complaints.

In analysing their material Byrne and Long write: "What stand out... is the degree to which any given consultation conducted by a particular doctor is like another consultation conducted by the same doctor" (p. 88). This typical way of managing patients they call the style of a doctor. But there was another striking feature: after the doctor during the consultations has taken a decision regarding the condition of the patient, he will often change styles rather drastically. "Once they have done their private decision-making they drop the style they are using and adopt a new style" (p. 90). With this, the importance of the distinction between the diagnostic phase and prescribing phase in a consultation has become manifest.

\section{Interview styles in the diagnostic phase}

Byrne and Long then first consider the diagnostic phase in which they distinguish four different styles of behaviour on the same dimension: doctor-centred behaviour-patient-centred behaviour. The more the style centres on the patient, the more the doctor will use the knowledge the patient has, allow the patient to contribute in the decision-making on what is wrong, etc. As the style, however, becomes more doctor centred, the doctor will be led to a greater extent by his own professional expertise, will determine the content of the discussion, etc. By far the most of the doctors studied by Byrne and Long used the styles 1 and 2 .

\section{Interview styles in the prescribing phase}

With the same four styles Byrne and Long hoped to cope with the prescribing phase. Bad luck. Byrne and Long write "Originally we tried to compress all our findings into four styles, but this only served to demonstrate that such a spectrum was inadequate to cover the range we were now concerned with. After various experiments we found that a range encompassing seven styles was the basic minimum we could use" (p. 104)* for the prescribing phase. The lowest numbered styles again show by far the highest incidence, in $65 \%$ of the investigated consultations style 1 or 2 prevailed.

It is to be regretted that Byrne and Long do not tell us more about the way in which this classification of styles arose, since the typology of styles is the basis for the second part of their research. On the ground of face validity they assigned the doctors to the various styles. They do not mention any problems with this arrangement; which is somewhat surprising as in our experience it is not always quite easy to distinguish related styles.

Having thus linked each style of behaviour to a specific type of interview, they take a further step in the analysis. For each of the 50 interview categories it is ascertained in which style of interview it has the highest incidence. In doing so they start from the idea that a particular category is likely to occur most

*Unfortunately Byrne and Long omit to tell us any further details. They to indeed emphasize here that this is a discrete ordinal scale. It is, therefore, cause for wonder that they lightly switch to a continuous scale later on. frequently in consultations of a particular style: the 'closed question' would be most frequent in highly doctor-centred consultations, 'reflecting' 'the use of silence' would mainly occur in patient-centred consultations, etc.

The next step to be taken is the "calculation" of the doctor/patient centredness of the consultation. On the basis of the frequency distribution over the various styles that is calculated for a specific category of expression (unit of speech), each category is assigned a certain weight. If, for example, an expression should occur mainly in style 4 , then this is valuated with mark 4. If a type of expression should occur frequently in several styles, such as 5, 6 and 7, then this unit is awarded the mean of these styles, i.e. 6 . In this way each category was rated for the diagnostic phase in a range from 1 to 4 inclusive, for the prescribing phase ranging from 1 to 7 inclusive. Apart from these, a cluster of negative behaviours was listed with the valuations -1 and -2 to show that such behaviour is inhibiting to the patient.

The calculation of the style score is then made as follows. For each doctor a frequency distribution of his verbal behaviours is designed over a large number of consultations. The number of units of verbal behaviour in each category is multiplied by the value of this category. The results are added up and divided by the total number of units and the result of this is a valuation representing which style, on the dimension doctor-centred vs patient-centred behaviour is that of the doctor involved.

Leaving aside that these assumptions appear rather suddenly in the book and have no empirical support, a few other sudden steps occur in the further elaboration. These result in unclarities and inconsistencies in the system which we shall revicw critically in the next section. After this appreciation-which rests on purely theoretical considerations-we shall further examine the question in how far the system nevertheless remains useful for our original objectives.

\section{FIVE CRITICISMS}

(1) Originally Byrne and Long used two discrete ordinal scales to describe the styles of the doctor. When they propose to calculate the style making use of all units of speech the reason for adopting a four point scale in the one stage and a seven point scale in the other have disappeared.

(2) Byrne and Long are also inconsistent in their use of the terms doctor-centred and patient-centred. Some categories with a low value are classified as patient-centred and some highly valued categories as doctor-centred (see Appendix).

(3) Neither are Byrne and Long systematic in their manner of assigning values to the categories. Most categories are given a certain value on the basis of their incidence in certain styles; a number of categories, though, i.e. the negative behaviours suddenly acquire absolute value, which moreover is equal for the four point scale and the seven point scalc (this gives a negative expression in the diagnostic phase a greater weight). How two categories can possibly get the value 0.5 remains in total darkness.

(4) In that they take the relative incidence in specific styles as the starting-point, various categories 
get a relative value in the diagnostic phase that differs from their value in the prescribing phase. This is obvious when we convert the four point scale of the diagnostic phase to a seven point scale. If we now express the category value both in the diagnostic phase and in the prescribing phase in a seven point scale, we see that with a number of categories, significant deviations in valuation occur (see the Appendix).

(5) Our final objection concerns the fact that, if the style of a GP is calculated by the valuations given to the units of speech by Byrne and Long, it is hardly possible for a doctor to get into the highest-valued style. There is but one category valued at 7: 'the doctor uses silence', and as a consequence a doctor would have to keep silent all the time to end up in style 7. Moreover, in any consultation a number of general remarks occur with an in-between value which pull the style rating of a consultation towards the mean value. As a consequence the styles as calculated no longer correspond to the original seven styles in the prescribing phase, and some obscurity results as to the exact meaning of a calculated style value. The same phenomenon is seen with the four styles of the diagnostic phase.

On account of these five criticisms we do not think it wise to continue working with the valuations of the categories and calculations of the interview style of a doctor as proposed by Byrne and Long.

\section{THE USEFUL ELEMENTS OF THE SYSTEM}

The critique formulated above is directed in particular against the assumption that a type of expression could be rated for its being doctor or patient-centred, and that it would therefore occur exclusively in the corresponding consultations. However, if we do not attempt to valuate the units of speech that make up the system of categories, this classification turns out to exhibit very helpful aspects. Below, we shall consider the reliability of the observation system, the practical applicability and the relevance of such a system.

For this purpose 36 consultations by six GPs from the NHI video store were watched by two independent observers. Every consultation is divided in one or more diagnostic phases. Each phrase uttered by the doctor that could be taken to be a self-contained unit of sense was assessed for its inclusion in an observation category. For the entire diagnostic phase the number of units included in any one category was counted up.

\section{Frequency distribution of the interview categories}

The Appendix gives an impression of the frequency with which the various categories are found in the diagnostic and prescribing phases: the figure represents the percentage of consultations in which the category involved was scored. There are only 15 categories occurring in more than half of the consultations. The majority of the differences between the diagnostic and prescribing phase is due to the fact that some units of behaviour can only be scored in the one phase and not in the other. If we leave out these differences, it is manifest that the same behav-
Table 1. Correlations between two observers with regard to a number of frequent categories

\begin{tabular}{lcc}
\hline Category number & $\begin{array}{c}\text { Diagnostic } \\
R_{\text {os } 1 \times \text { obs } 2}\end{array}$ & $\begin{array}{c}\text { Prescribing } \\
R_{\text {obs } 1 \times \text { obs } 2}\end{array}$ \\
\hline 3. Direct question & 0.74 & Inappl. \\
15. Repeating what patient said & 0.66 & Inappl. \\
$\quad$ for affirmation & & \\
16. Suggesting & $0.43^{*}$ & 0.70 \\
19. Directing & 0.75 & 0.66 \\
20. Giving information or opinion & 0.81 & 0.77 \\
21. Giving or seeking recognition & 0.79 & Inappl. \\
23. Broad question & 0.72 & Inappl. \\
25. Encouraging & 0.75 & Inappl. \\
27. Exploring & 0.87 & $0.40^{*}$ \\
28. Answering patient question & 0.74 & 0.77 \\
29. Accepting patient ideas & Inappl. & 0.66 \\
37. Indicating understanding & 0.87 & 0.81 \\
38. Advising & Inappl. & 0.74 \\
39. Terminating (direct) & Inappl. & 0.55 \\
41. Indirect terminating & Inappl. & $0.41^{*}$ \\
50. Rejecting patient offers & Inappl. & 0.56 \\
\hline
\end{tabular}

$* 0.001<\alpha<0.01$; the other coefficients: $\alpha<0.001$.

iours tend to occur frequently in both the diagnostic and the prescribing phase.

\section{Reliability}

Reliability concerns the extent to which independent observers arrive at the same interview profiles for a given consultation. For that purpose, for each consultation the frequencies of the separate interview categories tallied by the two observers have been compared to each other. The degree of conformity is expressed in a product moment coefficient for each category. Since the correlation between two observers makes little sense if the category itself is of rare occurrence, we only give the correlation coefficients that refer to the often recuring categories mentioned above (Table 1).

The categories of 'suggesting' (in the diagnostic phase), 'exploring' (in the prescribing phase), 'rejecting' and 'terminating' apparently are a source of possible disagreement. In view of the complicated comprehensive nature of the system of categories the other correlations are fairly satisfactory. Summarizing, we wish to confirm that in practice it appeared quite feasible to distinguish self-contained units of sense and to classify them. This was also done with satisfactory reliability, in the sense that independent observers achieve quite similar interview profiles of the same consultation. Any phrase or verbal behaviour could be classed in a category; and conversely, most categories appear to occur in over $10 \%$ of the consultations; the number of them that might in fact be redundant is slight. Incidentally, Byrne and Long, too, mention that narrowing down the system of categories was impossible. Practical applicability, however, is not the only requirement if the use of an instrument is to be decided upon. It must also have a certain relevance. By means of the following example we wished to illustrate the relevance of Byrne and Long's system of categories.

\section{Relevance: an example}

A consultation is concerned in which the discussion runs partially on a somatic, partially on a psychosocial subject and in which the differences as to type of interview style are obvious in the interview profiles. 


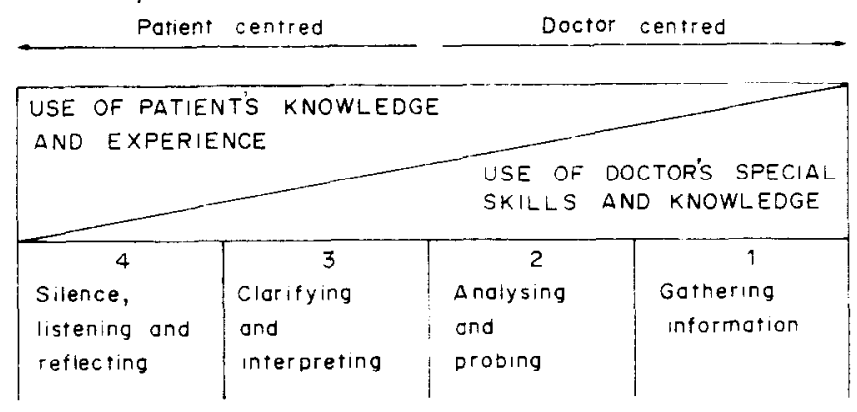

Fig. 1. Typology of styles in the diagnostic phase.

We shall first briefly discuss the contents of the consultation and thereupon present a few literal fragments from the consultation by way of illustration. Then we shall consider the interview profiles that arise if the consultation is scored according to the interview categories of Byrne and Long.

Consultation. The surgery is visited by a single man about 55 years old, who is an alcohol addict, but has now come for the pain in his legs. The man has been a resident of various institutes on account of his alcoholism and also has connections with the "A.A.Club'. On the request of the patient the doctor first examines the legs and then moves the discussion rapidly to his deteriorating condition. He tries to find out why the condition has deteriorated and what kind of help most appeals to the man at this moment. Finally the doctor advises him to re-establish contact with the 'A.A.-Club' on request he gives a prescription for the legs and asks the patient to return a week later.

From this consultation which lasted $10 \mathrm{~min}$ brief fragments have been taken to show how the discussion on the legs progresses (organic disease) and what the psycho-social part on the addiction looks like. The fragments have been split up in a diagnostic and a prescribing phase.

Figure 3 shows the interview profiles of this consul- tation, split up in diagnostic phase and prescribing phase and also in an organic disease part and a psycho-social part of the interview. For each category we indicate how often this behaviour occurred in the consultation, so absolute numbers are given. The interview profiles concern the whole conversation, not just the written fragments. The psycho-social part took more time than the organic disease topic, which also appears from the greater number of speech in the psycho-social part. Let us now turn to the data provided by the interview profiles.

The finding that stands out first is the greater variety in the verbal behaviour in the psycho-social fragments. In the purely somatic talk the doctor uses much less categories. This finding may bear some relation to what Byrne and Long refer to in their foreword: "We discovered that the doctors in our study appeared to have achieved set routines of interviewing patients, and that few of them demonstrated the capacity for variations of normal style and performance to meet the needs of those patients whose problems did not fit into an organic disease pattern" (p. 5). Making use of the interview profiles we may discover which doctors adopt different interview techniques for psycho-social complaints if compared to organic complaints and to what degree they do so.

\begin{tabular}{|c|c|c|c|c|c|c|}
\hline \multicolumn{4}{|c|}{ Patient centred } & \multicolumn{3}{|c|}{ Doctor centred } \\
\hline \multicolumn{7}{|c|}{$\begin{array}{l}\text { USE OF PATIENT'S KNOWLEDGE } \\
\text { AND EXPERIENCE }\end{array}$} \\
\hline 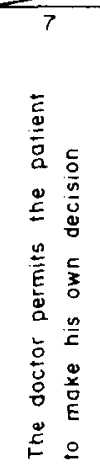 & 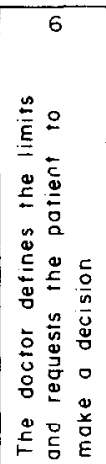 & 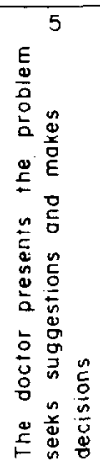 & 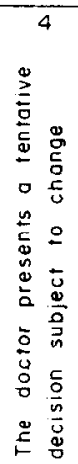 & 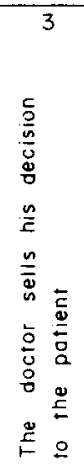 & 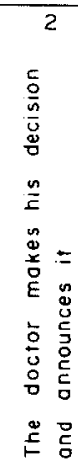 & 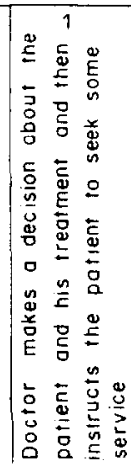 \\
\hline
\end{tabular}

Fig. 2. Typology of styles in the prescribing phase. 
Organic disease fragment diagnostic phase

Doctor: Well, come in (patient hobbles in)

What is it now?

Just take that chair for a while

Patient: I started drinking again, you may have read my reports already?

D: Yes, Yes...

P: $\quad$ But have a look here, this is becoming worse and worse (points to his legs), and even my feet and the other leg

D: What's becoming worse?

P: I' $m$ at my wits' end, really. I have been here to see your colleague doctor $\mathrm{X}$, those knee bandages, you know, they never worked properly. There is no solution that I know of. I bought the best shoes I could find, I don't know what to do next

D: What's wrong with your legs then?

P: Look, this is (points to legs) that gets blue all over

D: Yes, yes (looks). Does it hurt as well?

P: $\quad$ Yes, it hurts terribly, it ..

D: Are your feet cold too?

P: Often, and every morning this goes

D: Wrap them up again, will you (thereupon the doctor at once starts talking about the alcoholism)

Psycho-social fragment diagnostic phase

D: This doesn't look too well does it? (silence)

P: No, it's no good, if you read what's in all those

D: Yes.

P: What keeps worrying me ... that is I don't know what to do any more

D: What do you suggest?

P: $\quad$ Yes, well, I, I have been in A for half a year, have been in $\mathrm{B}, \mathrm{I}$ don't know what to do next

D: You can't leave drink alone? (silence)

P: It's so damned hard, I tell it as it is

D: Right, I know that, I do

P: I had my head examined, have gone to hospital A and B, I can show you (looks for letters)... It's such a wretched trouble, If I. .

D: What then, what is the wretched trouble?

P: (silence)

D: The alcohol... or the reasons for the alcohol?

P: $\quad$ There you have the trouble ... it has been wrong from childhood onward.

(The conversation continues in this way for about $6 \mathrm{~min}$ and finally the doctor asks what he can do for the patient. The patient then asks him to give something for the legs, which the doctor does and thereupon the doctor discusses what measures could be taken in respect of the alcoholism) reports from doctor $\mathrm{X}$

Organic disease fragment prescribing phase

D: And then I'll now give you something, and I hope it will help you, for because your liver does not properly work any more, you get blood-clotting disorders and I can't remedy that, but yet 1 hope it will be of some help

P: If only you make sure it is a combination that agree with well, eh, ... the alcohol

D: Yes, of course it does, right

P: $\quad$ For it's this, you see... you may prescribe as you do, but ...

D: Aspirin would not do, for instance

P: $\quad$ No it doesn't suit me any more

D: Fine

P: $\quad$ For I carry aspirin on me but I'll never use it

D: You mustn't do so either

P: $\quad$ For it's very dangerous

D: Yes

P: Because I already suffered from bleeding ulcers as well

D: Yes, that's why. Has another blood test been done on you?

P: $\quad$ Not yet

D: Shall we have one again?

P: Right, then I can go there at once, I don't mind at all. etc

(after this subject has been closed, the doctor refers once more to the addiction)

\section{Psycho-social fragment prescribing phase}

D: But what I would like to come back to. You yourself say that you halt between three opinions and that you can't make a choice

P: What ... It's not that easy

D: $\quad \mathrm{Mm}, \mathrm{mm}$... but as long as you don't make a choice there will be no improvement

P: $\quad$ Mm.mm ...yes, yes ... its not that easy

D: If it were easy you wouldn't be here

P: $\quad$ No, no... But I keep making demands on your time

D: Is that so bad?

P: $\quad$ Well, ah ... I beg your pardon, but if I, I, . . could only stop this action and reaction process ... ah ...

D: Yes, you'll have to go in a certain direction, you hang back from it

P: Yes, it's up and down all the time, you know... how should 1 say it

D: Yes, this circle, you got to break it, you got to break it, you must make up your mind for a decision

P: $\quad$ Right, it's kind of, yes, kind of a visual circle, yes

D: I think that's well seen. But yet, how can we guide you in finding an effective solution to your problem... I believe I'm in fact not able to do that ... but you do have those contacts with the Alcoholics Anonymous ...

P: $\quad$ Yes, I joined them and I'm certainly going to stay with them

(Finally the patient promises (hesitatingly) to go back to the 'A.A.-Club' and return next week)

Fig. 3

The interview profiles give hints as to the difference in content between psycho-social and organic disease interview fragments. In this consultation, for example, we see that the doctor takes a more 'Rogerian' attitude in the psycho-social part. Such as encouraging the patient more and indicating understanding of the patient's feelings. This is indeed the most obvious but not the only difference. The doctor, for instance, in this consultation, appears both to control and direct in the psycho-social fragment as well. This seems to indicate that just 'listening with understanding' is insufficient in the general practice, while the GP can play an active part in unraveling the problem and the subsequent discussion on the measures that may be taken also in respect of psychosocial complaints (see also [10]).

Which interview techniques could be adopted in such a case and what could be the effects may be studied with the aid of the interview profiles.

We finally have in mind the third possibility offered 
by the interview profiles. Since these profiles do discriminate so clearly between interview fragments on organic subjects and psycho-social subjects they cannot fail to be useful for revealing in what ways doctors can alter their interview behaviour. If, for example, a training course has been effective, this measuring instrument is sufficiently detailed to trace the changes in interview behaviour.

\section{CONCLUSION}

The system of categories proposed by Byrne and Long has proved to be of practical use as an instrument to describe in much detail the verbal behaviour of GPs. It is, however, too ambitious to pretend that the consultation may then be classified in a typology on the doctor/patient-centred dimension by means of a single figure, using the weights assigned to the various categories. On ground of a number of a priori objections as formulated, we tried to prove the plausibility of this. As a matter of fact, our objections were confirmed in practice when we calculated the style ratings for our 36 consultations by the formula evolved by Byrne and Long: the distribution was very narrow, and only a minimum of discrimination between doctor-centred and patient-centred consultations was obtained. Apart from these consultations, though, the instrument meets the requirements posed in the Introduction: it covers the entire consultation, it can reliably be used in practice, and the example we elaborated already shows that it has a certain relevance to general practice. Byrne and Long are in our opinion justified in stating that doctors have a style of their own in their conduct of consulting interviews. We recognized the same happening when we watched the video-taped consultations. If we now find that it is impossible-anyway if this approach is adoptedto express this style in one figure, we do not wish to
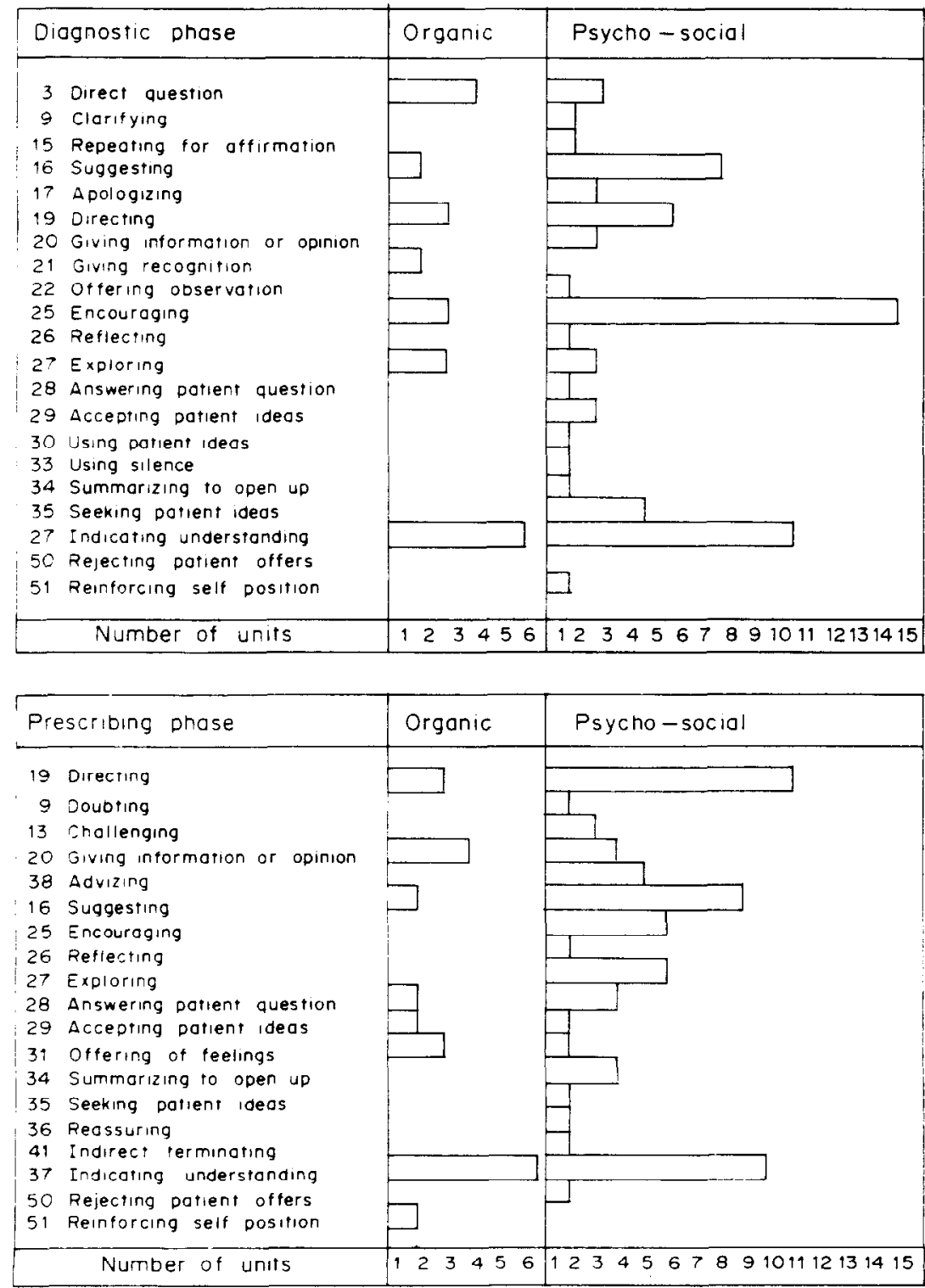

Fig. 4. Interview profiles: diagnostic prescribing phases, organic and psycho-social. 
throw the baby out with the bath water by ignoring that a doctor has a style of his own. The unweighted interview profile has, in our example, proved to be capable of distinguishing between typical styles - in this case not between doctors but between physical and psycho-social fragments. An increase in discriminating power may be obtained if the idea is abandoned that a doctor's style must be rated on one dimension only (doctor/patient-centred).

Some of the categories in the system aim predominantly at this dimension, and the scoring of these categories may serve to ascertain to what degree a doctor's work is patient-centred. Other categories may rather pertain to relations of power, empathy, giving information, etc. Further research will have to prove whether such relatively independent clusters do exist. If this should turn out to be the case, then the classification system will go to show for each dimension in how far it is present in the consultation. For this purpose the number of units of speech in the categories of one cluster is counted, and a calculation is made as to what proportion of the sum of all units in the consultation it occupies. By this procedure we can make an assessment as to the percentage units of speech expended on each dimension. A compressed interview profile arises in this fashion, consisting of as many ratings (figures) as there are dimensions. These figures are quite clear in what they signify, namely the percentage units of speech on each dimension. Thus, in our view, the observation system of Byrne and Long is used in a rather more legitimate way because as a result compressed interview profiles of doctors can be compared. (For that matter another study has also pointed out that the one dimensional character of the communication model of Byrne and Long is not satisfactory. Glynn Owen and Goodge [12] are in favour of a model in which directing and counseling coexist; their data suggest that those two dimensions appear independent of each other in consultations.) In this way the risk is avoided that differences in style (between doctors or dissimilar consultations) become concealed in one figure. It ensures that interview profiles may be estimated for a variety of dimensions, and that detailed feedback can be given. It prevents several problematic assumptions which Byrne and Long felt compelled to make in formulating their one-dimensional rating, while the effectiveness of the comprehensive classification system is put to the best use.

\section{REFERENCES}

1. Ekman P. and Friessen W. V. The repertoire of nonverbal behaviour; categories, origins, usage and coding. Semiotica 1, 49-98, 1969.

2. Ellgring J. H. Blickverhalten und Sprechaktivität: Untersuchungen zum sprachlichen und nichtsprachlichen Verhalten in zweipersonen Situationen. Dissertation, Marburg, 1975.

3. Schouwenburg H. C. Systematische observatie van huisartspatient interacties. Instituut voor Huisartsgeneeskunde, Amsterdam, 1978.

4. Katz E. Doctor-patient exchanges: a diagnostic approach to organisations and professions. Hum. Relat. 22, 309, 1969.

5. Bain D. J. G. The content of physician-patient communication in family practice. J. Fam. Pract. 8, 745-753, 1979.

6. Bain D. J. G. Doctor-patient communication in general practice consultations. Med. Educ. 10, 125-131, 1976.

7. Davis M. S. Variations in patients' compliance with doctor's advice: an empirical analysis of patterns of communication. Am. J. publ. Hlth 58, $274-288$.

8. Kent Smith C., Polis E. and Hadac R. R. Characteristics of the initial medical interview associated with patient satisfaction and understanding. J. Fam. Pract. 12, 283-288, 1981

9. Korsch B. M. and Negrete V. F. Doctor-patient communication. Scient. Am. 227, 66, 1972.

10. Bensing J. and Verhaak P. Ruimte voor de patient (Room for the patient). Ned. Tijdschr. Psychol. 37, 19-33, 1982.

11. Byrne P. S. and Long B. E. L. Doctors Talking to Patients. A Study of the Verbal Behaviour of General Practitioners Consulting in their Surgeries. HMSO. London, 1976.

12. Glynn Owen O. G. and Goodge P. Physiotherapists Talking to Patients. Patient Counseling and Health Education, Vol. 3, pp. 100-102, 1981 


\section{APPENDIX}

Units of Speech from Byrne and Long: Their Value in the Diagnostic and Prescribing Phase and Percentage Consultations in Which These Units Occur in the Diagnostic and Prescribing Phase

\begin{tabular}{|c|c|c|c|c|}
\hline \multirow[b]{2}{*}{ Doctor-centred behaviour } & \multicolumn{2}{|c|}{ Value in phase } & \multicolumn{2}{|c|}{ Occurrence in $\%$ consultations } \\
\hline & Diagn. & Prescr. & Diagnostic & Prescribing phase \\
\hline 1. Offering self & 1 & - & 19 & - \\
\hline 2. Relating to some previous experience & $1 \underline{1}$ & $1 \underline{\underline{t}}$ & 19 & 19 \\
\hline 3. Direct question & $1 \frac{1}{2}$ & - & 97 & - \\
\hline 4. Closed question & $\frac{1}{2}$ & - & 47 & - \\
\hline 5. Self answering question (rhetorical) & $i$ & - & 6 & - \\
\hline 6. Placing events in place/time/sequence & 2 & - & 42 & - \\
\hline 7. Correlational question & $1 \frac{1}{2}$ & - & 14 & - \\
\hline 8. Clarifying & 3 & $5 \frac{1}{2}$ & 47 & 39 \\
\hline 9. Doubting & $1 \frac{1}{2}$ & 3 & 31 & 19 \\
\hline 10. Chastizing & 1 & 2 & 14 & 14 \\
\hline 11. Justifying other agencies & 1 & 1 & 8 & 3 \\
\hline 12. Criticizing other agencies & 1 & 1 & 14 & 8 \\
\hline 13. Challenging & $2 \frac{1}{2}$ & 4 & 8 & 14 \\
\hline 14. Summarizing to close off & 1 & 2 & 6 & 33 \\
\hline 15. Repeating patient for affirmation & 2 & 3 & 67 & 36 \\
\hline 16. Suggesting & $1 \frac{1}{2}$ & 3 & 72 & 86 \\
\hline 17. Apologizing & $1 \frac{1}{2}$ & - & 19 & - \\
\hline 18. Miscellaneous professional noises & $1 \underline{1}$ & 1 & 19 & 19 \\
\hline 19. Directing & $1 \frac{1}{2}$ & $1 \frac{1}{2}$ & 64 & 92 \\
\hline 20. Giving information or opinion & 2 & 3 & 92 & 97 \\
\hline 38. Advizing & - & $3 \frac{1}{2}$ & - & 78 \\
\hline 39. Direct terminating & - & 1 & - & 64 \\
\hline 40. Suggesting or accepting collaboration & - & $4 \frac{1}{2}$ & - & 25 \\
\hline
\end{tabular}

\begin{tabular}{|c|c|c|c|c|}
\hline \multirow[b]{2}{*}{ Patient-centred behaviour } & \multicolumn{2}{|c|}{ Value in phase } & \multicolumn{2}{|c|}{ Occurrence in $\%$ consultations } \\
\hline & Diagn. & Prescr. & Diagnostic & Prescribing phase \\
\hline 21. Giving or seeking recognition & $1 \frac{1}{2}$ & - & 72 & - \\
\hline 22. Offering observation & 3 & 31 & 14 & 8 \\
\hline 23. Broad question & $2 \frac{1}{2}$ & - & 58 & - \\
\hline 24. Concealed question & 3 & - & 3 & - \\
\hline 25. Encouraging & 3 & $5 t$ & 78 & 44 \\
\hline 26. Reflecting & 4 & $6 \frac{1}{2}$ & 44 & 33 \\
\hline 27. Exploring & $2 \frac{1}{2}$ & $5 \frac{1}{2}$ & 72 & 69 \\
\hline 28. Answering patient questions & 2 & 4 & 61 & 86 \\
\hline 29. Accepting patient ideas & 4 & 6 & 33 & 53 \\
\hline 30. Using patient ideas & 3 & $4 \frac{1}{2}$ & 31 & 19 \\
\hline 31. Offering of feeling & $2 \frac{1}{2}$ & $4 \frac{1}{2}$ & 44 & 47 \\
\hline 32. Accepting feeling & 4 & 5 & 8 & 14 \\
\hline 33. Using silence & 4 & 7 & 14 & 6 \\
\hline 34. Summarizing to open up & 3 & $5 \frac{1}{2}$ & 31 & 19 \\
\hline 35. Seeking patient ideas & 4 & $4 \frac{1}{2}$ & 33 & 31 \\
\hline 36. Reassuring & $\mathrm{l}$ & 3 & 39 & 44 \\
\hline 37. Indicating understanding & $2+$ & 3 & 89 & 86 \\
\hline 41. Indirect terminating & - & 4 & _ & 72 \\
\hline 42. Pre-directional probing & - & $4 \frac{1}{2}$ & - & 14 \\
\hline
\end{tabular}

\begin{tabular}{lccccc}
\hline & \multicolumn{2}{c}{ Value in phase } & & \multicolumn{2}{c}{ Occurrence in \% consultations } \\
\cline { 2 - 3 } \cline { 5 - 6 } Negative behaviour & Diagn. & Prescr. & & Diagnostic & Prescribing phase \\
\hline 50. Rejecting patient offers & -1 & -1 & & 44 & 56 \\
51. Reinforcing self position & -1 & -1 & & 8 & 39 \\
52. Denying patient & -1 & -1 & & 3 & 8 \\
53. Refusing patient ideas & -1 & -1 & & 8 & 19 \\
54. Evading patient questions & -1 & -1 & & 8 & 8 \\
55. Refusing to respond to feeling & -2 & -2 & & 6 & 6 \\
56. Not listening & -1 & -1 & & 28 & 14 \\
57. Confused noise & (Scale value:) & $1-4$ & $1-7$ & & \\
\cline { 1 - 2 } & &
\end{tabular}

